\title{
A numerical simulation of methane-air mixture explosion under normal and elevated initial temperatures
}

\author{
$\mathrm{Na} \mathrm{Gao} \mathrm{*}$ \\ School of Chemical Engineering, Nanjing University of Science and Technology, Nanjing, Jiangsu \\ 210094, PR China \\ gaona_mxd@163.com
}

Keywords: Gas explosion, methane-air mixture, explosion temperature, initial temperature, flame propagation

Abstract. Study of the methane-air explosion mechanism is of great important for the safety utilization of the coal bed methane and safety production of coal mine. The methane-air explosion reactions were numerical simulated in a closed spherical $20 \mathrm{dm}^{3}$ vessel with the ignition electrode at the center. The simulation results show that the main areas of premixed gas are the burned zone, unburned zone and preheat zone, and the reactions take place in the preheat zone. The flame spreads to the wall with the reaction goes on. Higher initial temperature accelerates the reaction rate and the flame propagation velocity. When the initial temperature increases, the temperature of burned zone is raised more than the initial temperature, and the explosion temperature of methane-air mixture also increases.

\section{Introduction}

Flammable gas fuel was one of the most important energy sources in industry and daily life. [1]Coal bed methane $(\mathrm{CBM})$ is got more and more attention as a new clean energy. The main component of the CBM is methane. In the process of the exploitation, manufacture and use of the CBM, the risk mainly comes from the combustion and explosion of methane. The explosion accidents will take place due to the accidental ignition of premixed methane-air mixture, causing rapid pressure rise and high temperature which cause serious losses. $[2,3]$ As a kind of important energy, Coal still occupies an important position. Coal mine gas explosion accidents occurred frequently, which caused huge economic loss, casualties and a huge impact on the environment. Gas explosion is strong methane combustion reaction. The fires and explosions of the flammable gas loss more than other materials, [4-6] so it is important to get a clear understanding of the gas explosion mechanism, and thereby predicting and controlling accidents.

Explosion temperature is an important parameter of explosion reactions, which determines the reaction rate and affects the flame propagation. It is of great significance to understand the explosion mechanism. In this paper, on the basis of previous studies, explosion reactions of methane-air mixture were numerical simulated in a closed $20 \mathrm{dm}^{3}$ explosion vessel. By simulating methane-air explosion under the condition of different initial temperature, changes of explosion temperature in the reactions and the influence of the initial temperature on explosion temperature are analyzed, which provides certain theoretical basis for the safety utilization of the CBM and safety production of coal mine.

\section{Numerical simulation}

According to the flame propagation behaviors during methane-air explosions, and considering computing precision and computing time, $\mathrm{RNG} \mathrm{k}-\varepsilon$ model is selected as the turbulence model in this numerical calculation. In order to comprehensively consider the effect of turbulence and chemical kinetics of methane-air reaction, finite-rate combustion model is used to describe the turbulent combustion process. Two step reaction mechanism of methane combustion is taken to describe the reaction process, ignoring the middle material generated. 


\section{Reaction 1}

Reaction 2

$$
\mathrm{CH}_{4}+\mathrm{O}_{2} \rightarrow \mathrm{CO}+\mathrm{H}_{2} \mathrm{O}+Q_{1}
$$

$$
\mathrm{CO}+\mathrm{O}_{2} \leftrightarrow \mathrm{CO}_{2}+Q_{2}
$$

The reaction rate of the reaction 1 is mainly the control of reaction kinetics, while that of the reaction 2 mainly depends on turbulent intensity of the turbulent flow field. The control mechanism and energy release of the two steps are all different, but between the two there is a mutual coupling that affects the proceeding of the reaction. Furthermore, the reaction rate and temperature restrict mutually, which ensures that the reaction kinetics and turbulent flow control the methane-air explosion process together.

\section{Results and discussion}

Conditions of normal temperature. The premixed methane-air mixture is ignited in the center of the vessel, and then reactions start from the ignition point to the wall. The main areas of the vessel are the burned zone and unburned zone. The temperature of the burned zone is higher because of the exothermic reaction process. The temperature of unburned zone does not significantly increase, but with increasing reaction time, it elevate a bit as a result of the existence of thermal radiation, heat convection and so on. There is a preheat zone between these two areas, where combustible gas is heated to participate in chemical reactions under higher temperature. The temperature and reaction rate at $40 \mathrm{~ms}$ after ignition with the radius of the container at normal temperature and pressure are shown in Fig. 1. It can be seen that a narrow area of preheat zone near the burned zone is the main reaction area. Temperature has a great impact on the reaction rate, so the reaction rate is fast near the burned zone where the temperature is high, and there are no reactions in low temperature area.

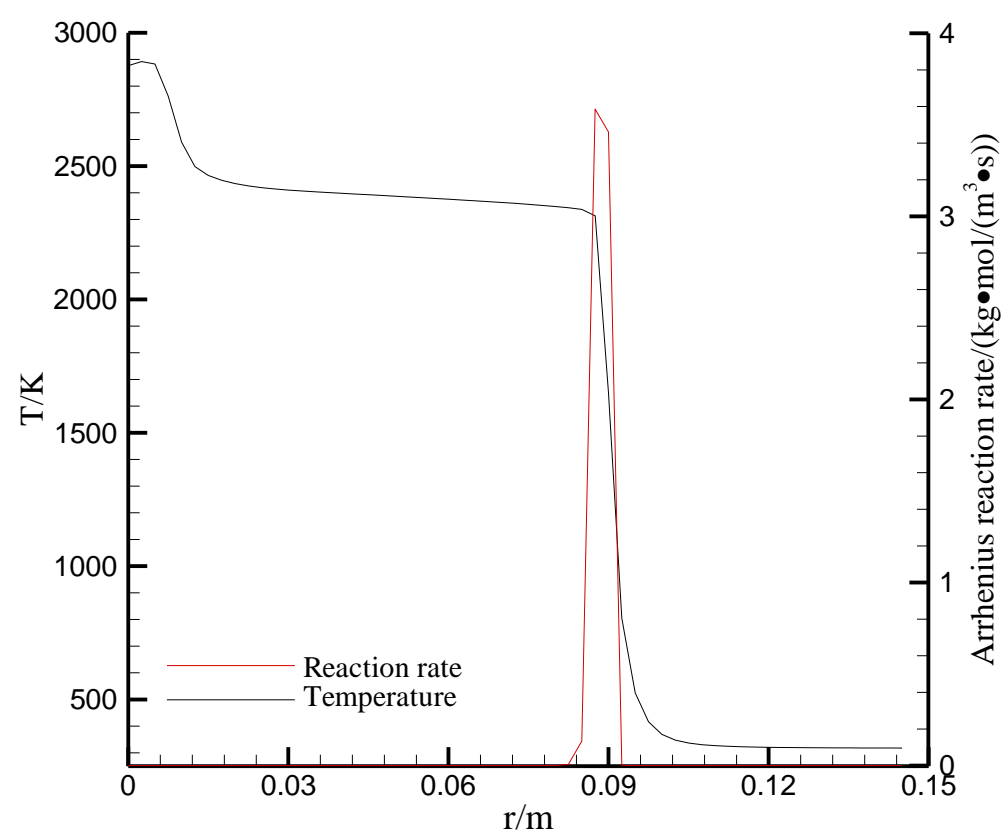

Fig. 1 Changes of temperature and reaction rate of methane-air mixture with the radius of the container at 40ms at normal temperature and pressure

As the reaction progress, the reaction zone moves from the ignition point to the wall, and the flame front also spreads forward with the reaction zone. Figure 2 shows the temperature distributions at different reaction times along with the radius direction at normal temperature and pressure. The temperature of burned zone is essentially the same in the early stage of the reaction, while changes a slightly higher in the late stage. The temperature of the burned zone increases with the process of reaction, and up to nearly $2700 \mathrm{~K}$ (around the methane-air explosion temperature) at $100 \mathrm{~ms}$. There is a high temperature zone near the ignition area. This is because that the temperature of this area soared into several thousands degree at the ignition moment, and 
reduces to around $3300 \mathrm{~K}$ after the ignition because of the endothermic reactions. There is less heat loss in this area because that the flame front spreads rapidly, so the temperature remains high in this area.

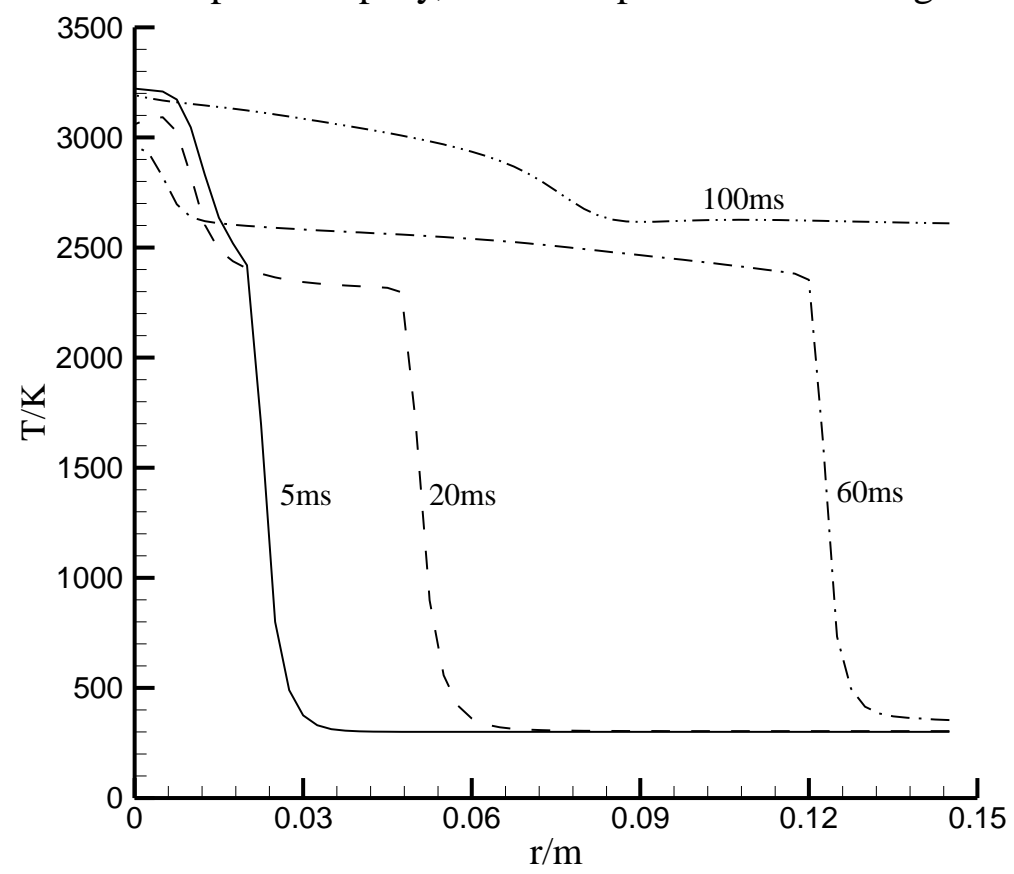

Fig.2 temperature at different reaction times with the radius direction at normal temperature and pressure

Conditions of elevated temperature. In the deep exploitation of coal mining, the gas is often in a state of high temperature and high pressure, what is more, secondary explosions are under the condition of high temperature and high pressure in gas explosions, so it has practical application value to study methane-air explosion reaction under special conditions. The reaction processes of methane-air mixture are simulated at elevated temperatures. As shown in Fig.3, it can be seen that at the same reaction time, the high-temperature burned zone is much wider under higher initial temperature, that is, the flame front spreads further. The temperature of burned zone increases with the initial temperature increases. When the initial temperature rises $100 \mathrm{~K}$, the burned zone temperature rises higher than $100 \mathrm{~K}$. It indicates that the influence of initial temperature on the initial stage of reaction temperature is not a simple linear superposition. Through comprehensive effects on reaction rate and turbulent flows, the initial temperature has a complex effect on the explosion process.

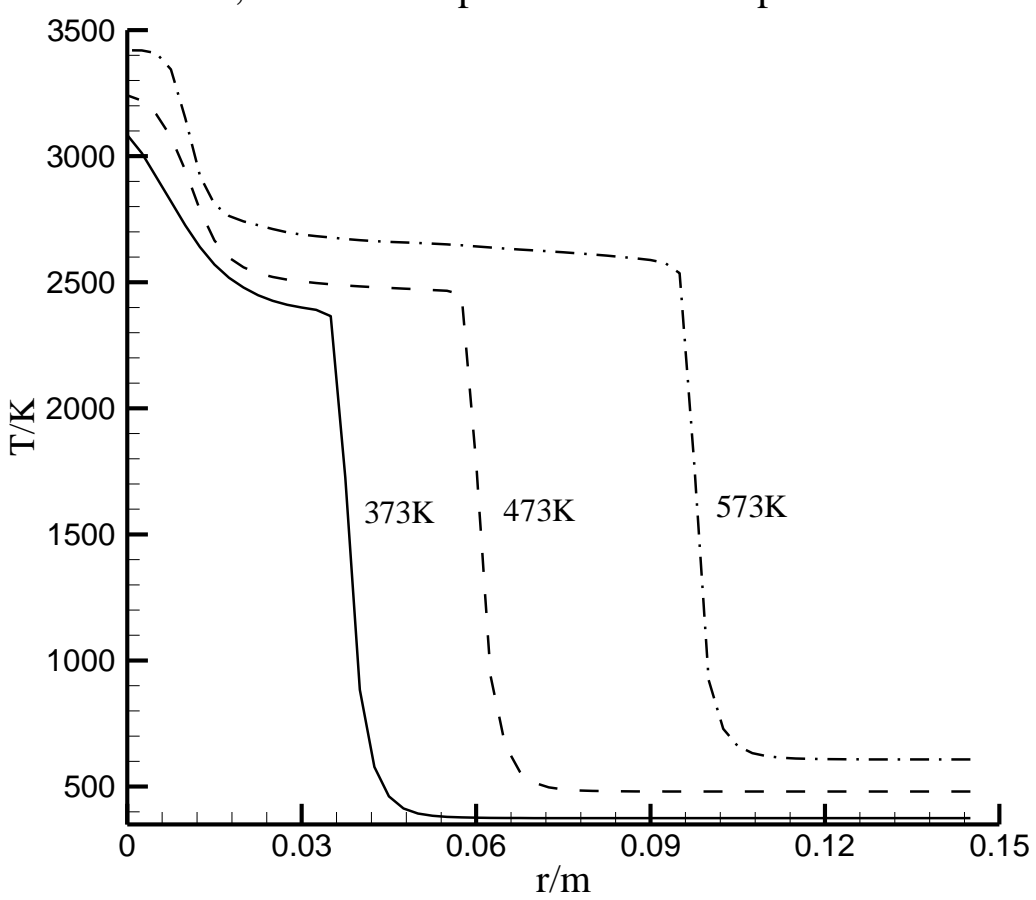

Fig.3 Changes of methane-air mixture explosion temperature at 10ms under different initial temperature 
Under the conditions of different initial temperature, the propagation velocity of flame is different, so rules of temperature at one point in the explosion vessel varies with the reaction time also change. Fig.4 shows temperature change curves of a point close to the wall under the initial temperature of $373 \mathrm{~K}, 473 \mathrm{~K}$ and 573 $\mathrm{K}$. Under the conditions of different initial temperature, the change rule of temperature varies with the reaction time at the same point remains basically the same. At the beginning of the reaction, the temperature remains essentially constant. It is preheated to a higher temperature with the reaction progress. When the reaction gets this point, the temperature dumps to one higher temperature, which is called explosion temperature. After the explosion, the temperature of this point basically remains unchanged. It can also be seen from Fig. 4 that the higher the initial temperature is, the higher the explosion temperature of methane-air mixture is.

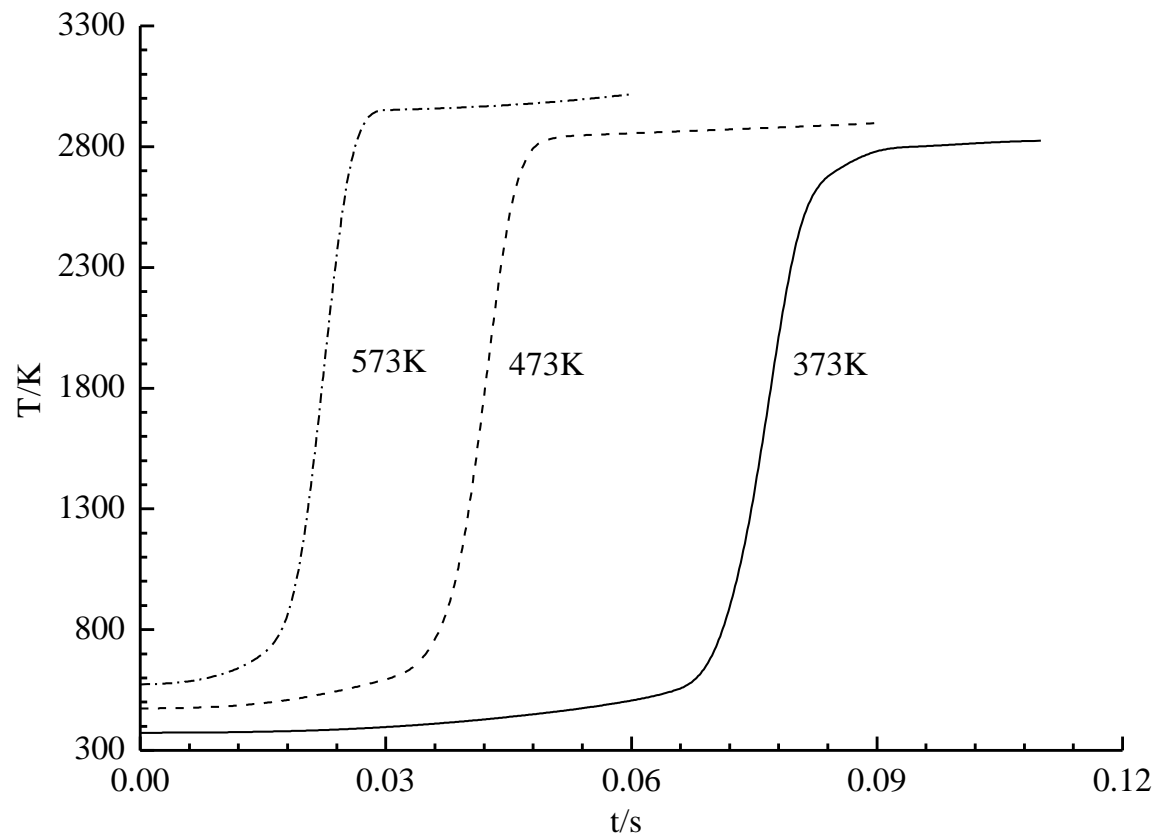

Fig.4 Changes of methane-air mixture explosion temperature under conditions of different initial temperature

\section{Conclusions}

There are the burned zone, unburned zone and preheat zone in methane-air mixture explosion. The chemical reactions take place at higher temperature area of the preheat zone, and the maximum reaction rate is got at the highest temperature. With the methane-air mixture explosion goes on, the reaction area moves to the wall, as well as the flame front.

The higher the initial temperature is, the wider the burned zone is, which means that higher initial temperature accelerates the flame propagation velocity. The temperature rise of burned zone is more than that of the initial temperature, as a result of influence of initial temperature on reaction rate and turbulent flows.

When the initial temperature varies, the rule of temperature change remains basically the same at the same point. The explosion temperature of methane-air mixture increases with the increase of initial temperature.

\section{References}

[1] Chen X F, Sun J H, Liu Y, et al. Chinese Sci Bull. 52(2007): 685-691.

[2] Gulder O L, Smallwood G J, Wong R, et al. Combust Flame. 120 (2000): 407-416.

[3] Trygve S, Diana C, Kjetil L O, et al. J Loss Prevent Proc. 30(2014):164-176.

[4] Yang Y, Liu J Z, Zhou T L, et al. Optically experimental study on flame microstructure and propagation of gas and dust explosion, Science Press, Beijing, 2004. 
[5] Dorofeev S B, Sidorov V P, Dvoinishnikov A E. Combust Flame. 104(1996): 95-110.

[6] Ratzed T K, Pantow E, Eichert H. Intern Assoc Hydr En-ergy. 21(1995): 407-414. 